\title{
Visual Receptive Field Heterogeneity and Functional Connectivity of Adjacent Neurons in Primate Frontoparietal Association Cortices
}

\author{
Pooja Viswanathan and ${ }^{-A n d r e a s ~ N i e d e r ~}$ \\ Animal Physiology, Institute of Neurobiology, University of Tübingen, 72076 Tübingen, Germany
}

The basic organization principles of the primary visual cortex (V1) are commonly assumed to also hold in the association cortex such that neurons within a cortical column share functional connectivity patterns and represent the same region of the visual field. We mapped the visual receptive fields (RFs) of neurons recorded at the same electrode in the ventral intraparietal area (VIP) and the lateral prefrontal cortex (PFC) of rhesus monkeys. We report that the spatial characteristics of visual RFs between adjacent neurons differed considerably, with increasing heterogeneity from VIP to PFC. In addition to RF incongruences, we found differential functional connectivity between putative inhibitory interneurons and pyramidal cells in PFC and VIP. These findings suggest that local RF topography vanishes with hierarchical distance from visual cortical input and argue for increasingly modified functional microcircuits in noncanonical association cortices that contrast V1.

Key words: functional connectivity; prefrontal cortex; single-unit recordings; ventral intraparietal

Significance Statement

Our visual field is thought to be represented faithfully by the early visual brain areas; all the information from a certain region of the visual field is conveyed to neurons situated close together within a functionally defined cortical column. We examined this principle in the association areas, PFC, and ventral intraparietal area of rhesus monkeys and found that adjacent neurons represent markedly different areas of the visual field. This is the first demonstration of such noncanonical organization of these brain areas.

\section{Introduction}

The receptive field (RF) of a neuron, the region of visual space from which its response can be modulated, is one of the basic concepts in vision research (Hubel and Wiesel, 1962). In the primary visual cortex (V1), a visuotopic map for points in the visual field arises from the ordered spatial arrangement of thalamic afferent axons in the cortex (Kremkow et al., 2016). Therefore, adjacent neurons within functional units lying perpendicular to the cortical surface (modules, microcolumns) show nearly identical RF locations (DeAngelis et al., 1999). This organization principle is commonly assumed to be realized even in the high-level association cortices of the frontal, parietal, and temporal lobes so that all neurons within a given microcolumn share the representation of a region of visual field (Suzuki and Azuma, 1983; Wilson et al., 1994; Rosa, 1997).

Received March 27, 2017; revised June 6, 2017; accepted July 13, 2017.

Author contributions: P.V. and A.N. designed research; P.V. performed research; P.V. analyzed data; P.V. and A.N. wrote the paper.

This work was supported by Deutsche Forschungsgemeinschaft Grant NI 618/2-1 to A.N.

The authors declare no competing financial interests.

Correspondence should be addressed to Dr. Andreas Nieder, Animal Physiology, Institute of Neurobiology, Auf der Morgenstelle 28, University of Tübingen, 72076 Tübingen, Germany. E-mail: andreas.nieder@uni-tuebingen.de. DOI:10.1523/JNEUROSCI.0829-17.2017

Copyright $\odot 2017$ the authors $\quad 0270-6474 / 17 / 378919-10 \$ 15.00 / 0$
We tested this prediction and investigated visual RF characteristics of adjacent neurons recorded at the same electrode tip in two association brain areas, the ventral intraparietal area (VIP) in the posterior parietal cortex, and the lateral PFC. The posterior parietal cortex is the termination zone of the dorsal, occipitoparietal visual stream that conveys motion and spatial information from V1 via MT/MST to VIP (Mishkin et al., 1983; Kravitz et al., 2011), which, in turn, is reciprocally connected with the PFC at the apex of the cortical hierarchy (Lewis and Van Essen, 2000). In agreement with such strong visual inputs, neuronal responses to visual parameters of stimuli are readily found in both the VIP (Colby et al., 1993; Duhamel et al., 1998; Chen et al., 2014) and the PFC (Goldman-Rakic, 1995; O'Scalaidhe et al., 1997; Zaksas and Pasternak, 2006), in addition to multimodal and executive function-related signals (Fuster, 2000; Miller and Cohen, 2001; Avillac et al., 2005, 2007; Schlack et al., 2005; Sugihara et al., 2006; Nieder, 2012; Guipponi et al., 2013). Moreover, the VIP and PFC are key nodes of the parietofrontal network processing numerical information (Nieder, 2016; Ramirez-Cardenas et al., 2016). Physiological parameters, such as selectivity latencies to quantity stimuli and categorical robustness, suggest that number information is processed hierarchically between both areas (Viswanathan and Nieder, 2013; Jacob and Nieder, 2014). Whereas VIP seems to be the first cortical hub extracting quantitative information, 
the PFC is a putative recipient of information about numerosity. Insights into the visual RF characteristics and wiring patterns of adjacent neurons in the VIP and PFC could elucidate their different roles in numerical processing. Indeed, we find considerable incongruencies in the spatial layout and position of RFs of adjacent neurons, suggesting RF organization that differs not only from that of early visual cortex, but also between these areas of the frontal and parietal association cortex.

Differences in the spatial tuning properties of adjacent neurons suggest distinct neuronal connectivity patterns in association cortices compared with V1. The microcircuitry of V1, formed by dedicated excitatory and inhibitory neurons, is considered canonical and is thought to generalize to other neocortical regions (Douglas and Martin, 2004). For instance, inhibitory interneurons in local microcircuits play a major role in shaping and often, sharpening neuronal response properties of excitatory pyramidal projection neurons (Markram et al., 2004). However, whether brain areas that are higher up the visual hierarchy and display noncanonical circuit properties enabling parallel and reentrant processing required for cognition (Goldman-Rakic, 1988), exhibit the same basic principles remains unknown. We therefore characterized the functional connectivity between adjacent neurons and compared it with the observed RF heterogeneity in VIP and PFC. To identify putative inhibitory interneurons and pyramidal cells in extracellular recordings, we exploited established action potential waveform differences found in PFC between pyramidal cells that tend to exhibit broad action potential waveforms and interneurons that display narrow action potential waveforms (Wilson et al., 1994; Rao et al., 1999; Johnston et al., 2009; Merchant et al., 2012). By comparing the functional connectivity patterns between these two classes of neurons that constitute members of local microcircuits, we identified differential functional interactions that correlated with RF incongruences in VIP and PFC.

\section{Materials and Methods}

Experimental setup. Recordings were made in two male rhesus monkeys (Macaca mulatta) weighing between 5.5 and $6.3 \mathrm{~kg}$. The monkeys sat in primate chairs positioned $57 \mathrm{~cm}$ from a 15 inch flat-screen monitor within chambers. The monitor had a resolution of $1024 \times 768$ pixels and a refresh rate of $75 \mathrm{~Hz}$. We used the National Institute of Mental Health Cortex program to present the stimuli, monitor the behavior, and collect behavioral data. An infrared tracking system (ISCAN) was used to monitor the monkeys' eye movements. All data analysis was performed using the MATLAB computational environment (The MathWorks) using custom-written scripts. All experimental procedures were in accordance with the guidelines for animal experimentation approved by the national authority, the Regierungspräsidium Tübingen, Germany.

Behavioral task. Monkeys performed a passive fixation task during the $\mathrm{RF}$ measurements. While monkeys fixated a central white square $\left(0.10^{\circ} \times\right.$ $0.10^{\circ}$ of visual angle or dva) and maintained their gaze within $1.75 \mathrm{dva}$ of the fixation point, a light gray moving bar $\left(3^{\circ} \times 0.20^{\circ}\right)$ appeared on the screen at a pseudo-randomly chosen position from a $10 \times 8$ matrix of positions (see Fig. 1A). Each position was sampled for $1000 \mathrm{~ms}$ first with the bar oriented vertically moving left to right $\left(0^{\circ}\right.$ for $\left.250 \mathrm{~ms}\right)$, then right to left $\left(180^{\circ}\right.$ for $\left.250 \mathrm{~ms}\right)$, oriented horizontally moving up ( $90^{\circ}$ for 250 $\mathrm{ms})$, and moving down $\left(270^{\circ}\right.$ for $\left.250 \mathrm{~ms}\right)$. Then the bar was displayed at another location in the grid and three other locations after that, thus scanning 5 locations in every trial. The matrix of locations thus covered an area $30.5^{\circ} \times 23^{\circ}$ of central vision, centered on the fovea. The monkeys fixated the central fixation spot for the entire period of the trial and were rewarded for successful fixation. Each RF block consisted of 3 trials per position. The RF blocks were interspersed with blocks of a delayed match-to-sample task during which numerosities were presented in the center of the screen (Viswanathan and Nieder, 2013, 2015). We collected up to 4 blocks of RF trials per recording session.
Surgery and neuronal recordings. Both monkeys were first implanted with a head bolt with which we fixated the head to allow eye movements to be monitored during the task. We then implanted recording chambers over the right dorsolateral PFC, centered on the principal sulcus, and the right intraparietal sulcus guided by anatomical MRI of individual monkeys and stereotaxic measurements (see Fig. 1B). The surgeries were performed under sterile conditions while the monkeys were under general anesthesia. They received antibiotics and analgesics after the procedure.

We recorded neuronal signals from the 2 monkeys using arrays of eight glass-coated tungsten microelectrodes (Alpha Omega) for each area attached to screw microdrives in a grid with $1 \mathrm{~mm}$ spacing. Once the microelectrodes were lowered into the recording position, they were allowed to rest in the position for 30-60 min before recording began. The microelectrodes were not moved further during the recording session. VIP recordings were made exclusively at depths of $9-13 \mathrm{~mm}$ below the cortical surface. As has been reported previously, we observed visual and somatosensory responses on VIP electrodes. However, the neurons recorded were not preselected online for any sensory, spatial, or taskrelated parameter. The electrophysiological signals were amplified and filtered, and waveforms of the actions potentials sampled at $40 \mathrm{kHz}$ from each electrode were stored (Plexon Systems). Sorting of single units was performed offline based on waveform characteristics (Offline Sorter, Plexon Systems). Timestamps of trial events and action potentials were extracted for analysis.

Analysis of spatial selectivity (RF mapping). We analyzed the neuronal activity during the presentation of the moving bar stimulus to test selectivity of single neurons. To account for the well-known differences in response latencies in these two areas (Nieder and Miller, 2004; Viswanathan and Nieder, 2013), we delayed the onset of the $1000 \mathrm{~ms}$ analysis window for VIP neurons by $50 \mathrm{~ms}$, and for PFC neurons by $100 \mathrm{~ms}$. The selectivity of single neurons was tested using a three-way ANOVA $(p<$ 0.05) (Rainer et al., 1998; Romero and Janssen, 2016) on firing rates calculated in $250 \mathrm{~ms}$ analysis windows (corresponding to the duration of a single bar sweep) with position, movement direction, and orientation of the bar as factors. The movement direction was a nested variable of orientation as the direction of movement depended on the orientation of the bar in that position. We downsampled the screen into five zones (top left, top right, bottom left, bottom right, and center) to reduce the number of false-positives from testing each of the 80 positions individually. Repeating the ANOVA with the zones defined in different ways yielded qualitatively and quantitatively similar results. We tested every neuron with a minimum of 10 trials per zone ( 859 PFC neurons and 693 VIP neurons).

We created raw RF maps for single neurons by averaging the responses over the entire $1000 \mathrm{~ms}$ period of visual stimulation at each position and then over all the trials at that position. As the moving bar covered an area of $\sim 3^{\circ} \times 3^{\circ}$ in the two different orientations, we linearly interpolated this $10 \times 8$ map by threefold interpolation in both spatial dimensions (Rainer et al., 1998) to visualize the responses at a resolution of 0.4 dva. We smoothed these with a 2D Gaussian kernel of 2 dva. These steps were taken to create smooth high-resolution RF maps that could be compared with those reported in other studies using large or sparse stimuli, with minimal assumption about the size or shape of the observed RF.

For neurons selective to the factor position in the ANOVA, we conducted a further cross-validation to confirm robust spatial selectivity. To that aim, we created two separate RF maps for each neuron: one from the first half of RF trials and another from the second half of RF trials. We calculated a 2D cross-correlation between the two maps created for each neuron and compared this against a distribution of 2D cross-correlations calculated from 1000 shuffles of each half map as follows:

$$
r=\frac{\sum_{h} \sum_{v}(\text { Half } 1-\overline{\text { Half } 1})(\text { Half } 2-\overline{\text { Half } 2})}{\sqrt{\left(\sum_{h} \sum_{v}(\text { Half } 1-\overline{\text { Half } 1})^{2}\right)\left(\sum_{h} \sum_{v}(\text { Half } 2-\overline{\text { Half } 2})^{2}\right)}}
$$

where Half 1 is the map created from the first half of trials and Half 2 is the map created from the second half of trials. The means of the maps are subtracted before summing them over the horizontal $(h)$ and vertical $(v)$ dimensions. Only if the true correlation across halves of trials lay above 
A

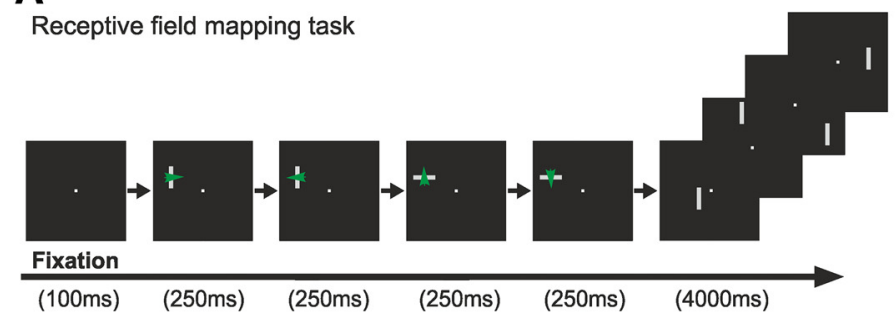

B

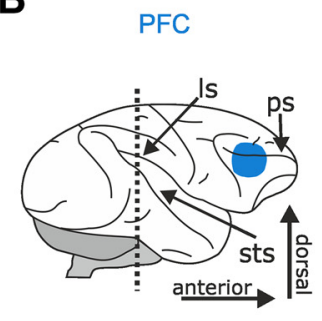

VIP

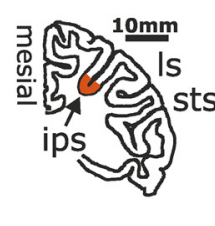

Figure 1. RF mapping in PFC and VIP.A, Passive fixation task. Monkeys fixated a central fixation target while a bar $\left(3^{\circ} \times 0.20^{\circ}\right)$ moving in four directions appeared in five successive locations on the screen. The visual field on the screen was divided into a grid of 80 locations ( $10 \times 8)$ sampled over 16 trials/cycle. $\boldsymbol{B}$, Lateral view (left) of a macaque monkey brain and a sagittal section (right) of the intraparietal sulcus at the position indicated by the dotted line. Positions of recording sites in the dorsolateral PFC (cyan) and the VIP (orange) in the depth of the sulcus are shown. ps, Principal sulcus; Is, lateral sulcus; ips, intraparietal sulcus; sts, superior temporal sulcus.

the 95th percentile of the distribution of surrogate correlations (onetailed, $p<0.05$ ), we accepted the neuron and its RF map into further analysis.

Analysis of neuronal pairs. We identified spatially selective neurons that were recorded from the same electrode with some temporal overlap and, thus, at the same location ("adjacent neurons"). To quantify the spatial similarity of their RFs, we calculated a $2 \mathrm{D}$ cross-correlation between their raw RF maps (DeAngelis et al., 1999). We did this to have a similarity index comparable across neuron pairs without assuming the shape or size of the RFs and have a measure that is sensitive to shifts of RFs from neuron to neuron in both dimensions as follows:

$$
r=\frac{\sum_{h} \sum_{v}(\text { Neuron } 1-\overline{\text { Neuron } 1})(\text { Neuron } 2-\overline{\text { Neuron } 2})}{\sqrt{\begin{array}{l}
\left(\sum_{h} \sum_{v}(\text { Neuron } 1-\overline{\text { Neuron } 1})^{2}\right) \\
\times\left(\sum_{h} \sum_{v}(\text { Neuron } 2-\overline{\text { Neuron } 2})^{2}\right)
\end{array}}}
$$

where Neuron 1 is the RF map of one of the neurons in the pair and Neuron 2 is the RF map of the second neuron in the pair. The means of the maps are subtracted before summing them over the horizontal $(h)$ and vertical $(v)$ dimensions. We additionally examined the contributions of the horizontal and vertical dimensions separately to the similarity between neuronal pairs.

We compared the derived true correlation coefficient value of the pair against the distribution of 1000 surrogates obtained from shuffling their maps. If the true coefficient lay above the 97.5th percentile of the shuffled distribution, we judged the neuronal pair to be congruent (two-tailed, $p<0.05$ ). If it lay below the 2.5 th percentile of the shuffled distribution and $<0$ (negative), we judged these maps to be inverted. If the true correlation lay within the shuffled distribution (2.5th and 97.5 th percentiles), we judged these maps to be incongruent (see Fig. 3).

Classification of cell types using waveforms. We classified all the recorded single units into narrow-spiking (NS) and broad-spiking (BS) neurons based on their waveforms (Diester and Nieder, 2008; Viswanathan and Nieder, 2015; Jacob et al., 2016). We saved the template waveforms for each single unit, sampled at a frequency of $40 \mathrm{kHz}$ (one entry every $25 \mu \mathrm{s}$ ), and only included the waveforms with a trough followed by a crest after reaching the threshold voltage (1951 of 2130 neurons). The troughs of the waveforms were expected to occur within 200-400 $\mu$ s of crossing the threshold voltage and the crests only after $300 \mu$ s of reaching threshold. To maximize the strength of our clustering procedure, we used all the neurons recorded across both areas. We normalized each waveform to the difference between the maximum amplitude and the minimum amplitude and aligned them to their troughs. We then entered the waveforms through a linear classifier ( $k$ means; $k=2$, squared Euclidian distance) to cluster the cells into two categories: NS and BS such that, on average, the units with smaller widths constituted the NS cluster (537 neurons) and those with the larger widths constituted the BS cluster (1414 neurons) (see Fig. 4A,B).

Cross-correlation between spike trains of neuron pairs. We computed a cross-correlogram by accumulating spike occurrence times in one neu- ron relative to the spikes of the paired neuron to identify functionally coupled neurons (Aertsen et al., 1989; de Oliveira et al., 1997; Diester and Nieder, 2008) among each pair of neurons that were recorded simultaneously with significant temporal overlap. To increase the number of action potentials, we included trials of the delayed match-to-sample task for this calculation. In addition, we repeated this analysis for a subset of neuronal pairs with sufficiently high firing rate using only trials from RF mapping to confirm that connectivity was independent from task demands. For each pair, one unit was assigned the trigger (in the case of NS-BS pairs, always the NS neuron) and for each spike recorded from this unit, the time delays to each of the spikes of the other unit were plotted in a histogram of $\pm 50 \mathrm{~ms}$ delay and a bin width of $1 \mathrm{~ms}$. Repeating this procedure for all spikes and all trials yielded the raw crosscorrelogram. We normalized the raw cross-correlogram by calculating a $z$ score (i.e., subtracting the mean and dividing by the SD), rendering it independent from the firing rates of the two units (de Oliveira et al., 1997). To avoid false-positives from short fluctuations, we smoothed the $z$ score with a 3-point boxcar ( $3 \mathrm{~ms}$ ). $z$ scores within $\pm 25 \mathrm{~ms}$ of the 0 th bin that crossed \pm 2.5 were considered significant to reject the null hypothesis that the correlogram at that point arose from two independent random Poisson processes. We also calculated a shifted predictor by correlating the trigger spike with the other neuron's response in the subsequent trial and the last trial with the first. We calculated the $z$ score of the shifted predictor as well to compare against the true correlogram and ensure significant deflections in the true correlogram were not influenced by other repetitive features, such as the stimulus onset, but truly reflected functional connectivity. Only those pairs with at least 1000 entries (i.e., action potentials or trials) for the correlogram were evaluated. When we included trials from the delayed match to sample task, these were 128 of 276 pairs total. Using only RF mapping trials, we could evaluate the correlograms of 61 pairs.

\section{Results}

\section{Visual RFs in PFC and VIP}

We mapped the RFs of a total of 859 neurons in PFC (361 from Monkey L and 498 from Monkey S) and 693 neurons in VIP (273 from Monkey L and 420 from Monkey S) with bars moving in different directions that were presented in systematically arranged locations on a screen (Fig. $1 A, B$ ). Many neurons showed spatially selective responses to the stimuli at confined locations of the visual field. The activity of an example neuron from PFC is shown in Figure $2 A-C$, and an example neuron from VIP is shown in Figure $2 D-F$. The detailed discharges to the different bar locations in the grid are depicted as dot-raster histograms (Fig. $2 A, D$ ) and averaged as spike-density histograms (Fig. 2B,E). To statistically verify neuronal selectivity, the firing rates elicited by the visual stimuli were tested using a three-way ANOVA (with position, direction, and orientation of the stimulus as factors; $p<$ $0.05)$. Only spatially selective neurons with a main effect for position were considered for further analyses. In PFC, 64\% (545 of 859) of the neurons showed a stimulus position effect, whereas 
A

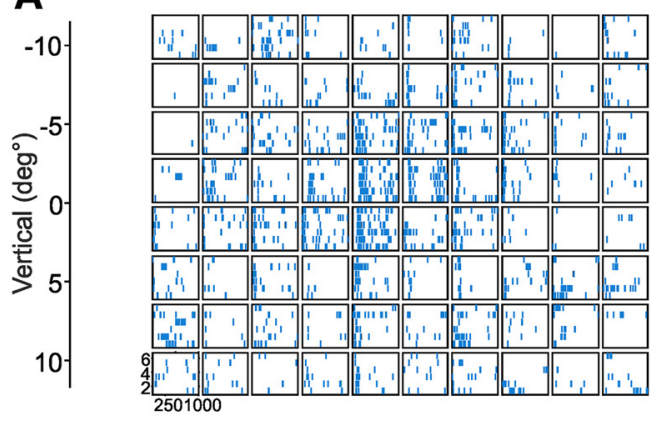

D

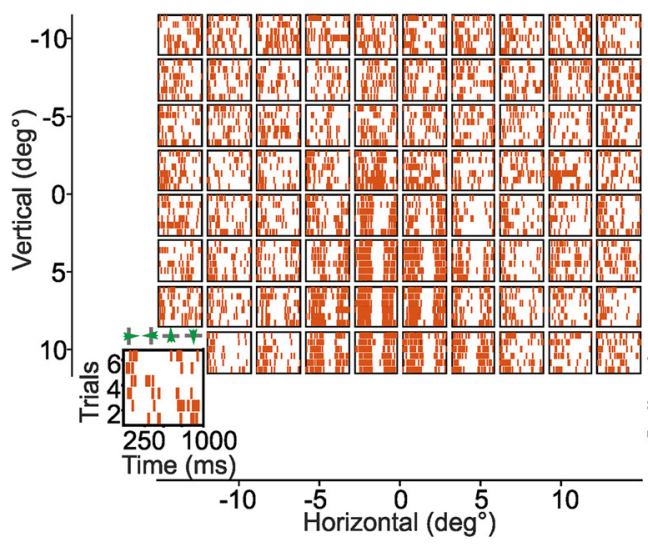

B

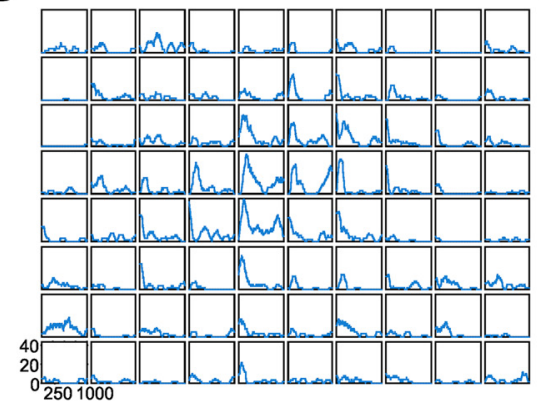

$\mathbf{E}$

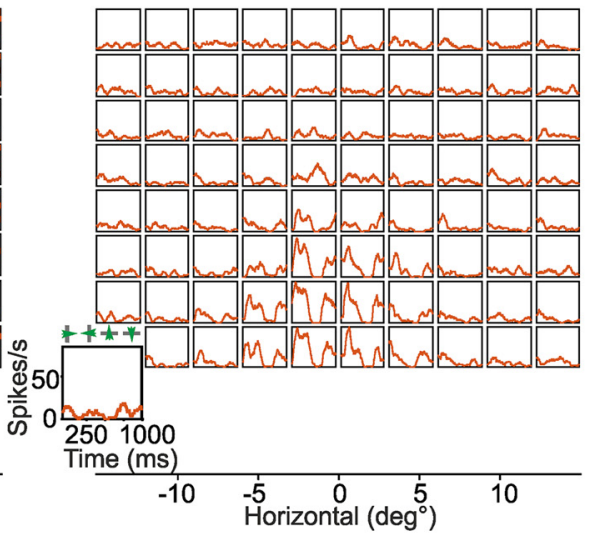

C
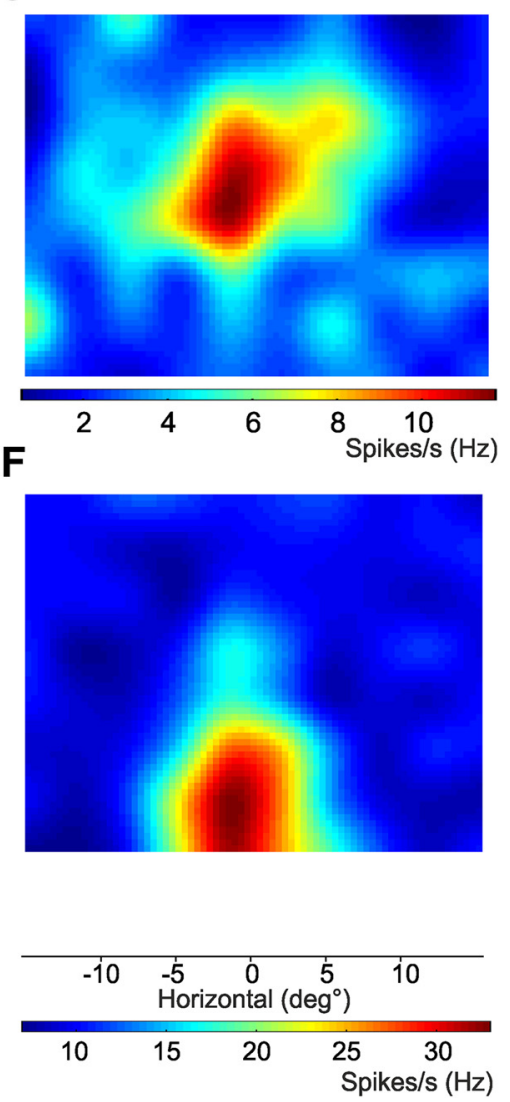

Figure 2. Example neurons with RFs. $\boldsymbol{A}$, Raster plot of an example spatially selective PFC neuron recorded in Monkey S. Each of the 80 locations is represented by a subplot, within which the dots along each line indicate the action potentials elicited for each trial. $\boldsymbol{B}$, Peristimulus time histogram for the same neuron as in $\boldsymbol{A}$. Each location is again represented by a subplot where the colored line indicates the trial-averaged response of the neuron in time. C, Averaged and smoothed high-resolution RF map ("heat map") for the neuron. Colors represent the firing rates across the measured visual field. $\boldsymbol{D}-\boldsymbol{F}$, The same plots as $\boldsymbol{A}-\boldsymbol{C}$ for an example neuron recorded in VIP from Monkey L. Enlarged bottom left squares represent the scale of each subplot of $\boldsymbol{A}, \boldsymbol{B}, \boldsymbol{D}$, and $\boldsymbol{E}$. The moving bar moved in four different directions within each position of the grid.

$69 \%$ (480 of 693 ) of the VIP neurons were found to be spatially tuned (see Materials and Methods).

From these spatially arranged discharges, we created averaged and smoothed RF maps (activity "heat maps" Fig. 2C,F) for each spatially tuned neuron. To further verify robust and reliable spatial tuning of single cells, we cross-validated the RFs of all ANOVA-selective neurons. For each neuron, two separate RF maps for the discharges to the first and second half of the trials were created. A 2D correlation analysis was applied to the two maps from a given neuron, and the resulting true correlation coefficient was compared with a distribution of coefficients obtained from shuffled surrogates. If the true correlation coefficient was significantly higher than the shuffled coefficients (one-tailed, $p<0.05$ ), the neuron was classified as spatially tuned and further analyzed. In the PFC, 50\% (425 of 859) of all recorded neurons were spatially selective according to both ANOVA and crossvalidation analyses. An even higher proportion of 57\% spatially selective neurons (396 of 693) was found in the VIP. This difference in the proportion of spatially tuned neurons between PFC and VIP was significant $\left(\chi^{2}=9.05, p<0.01\right)$. Some of the neurons displayed complex RFs, but many showed local, uniform RFs centered on the fovea or more contralaterally located, matching the early reports of visual or memory fields in PFC (Mikami et al., 1982; Rainer et al., 1998) and the reports of eye-centered RFs in VIP (Chen et al., 2014).

\section{Heterogeneous spatial RFs despite anatomical proximity} Anatomically nearby neurons in early visual cortex show spatially congruent RFs (DeAngelis et al., 1999), pointing toward an orderly local topographic arrangement of visual space. However, when we inspected RFs from adjacent neurons in PFC and VIP, unexpected RF incongruencies surfaced. We therefore analyzed spatial tuning of adjacent neurons in PFC and VIP. To ensure that neurons were anatomically adjacent, we analyzed neuron pairs that were recorded simultaneously or with some temporal overlap at a given electrode tip and separated based on differential waveform characteristics (off-line spike sorting). Because such neuron pairs were recorded at the same electrode tip, they were at the closest possible anatomical location. We found 127 such adjacent-neuron pairs in PFC (41 from Monkey L and 86 from Monkey S) and 149 pairs in VIP (24 from Monkey L and 125 from Monkey S) consisting of 131 unique neurons in PFC and 148 neurons in VIP. As can be seen in Figure 3, RF congruency was variable between neuron pairs, ranging from almost identical RFs with excitatory hotspots at corresponding visual field locations (Fig. $3 A, B$ ), to clearly dissimilar locations of excitation (Fig. $3 C, D)$ and, finally, RFs in which excitatory and inhibitory subfields seemed opposite in one neuron of a pair relative to the other (Fig. $3 E, F$ ).

To quantify the similarity or dissimilarity between the RFs of neuron pairs in an unbiased way that accounted for the different 

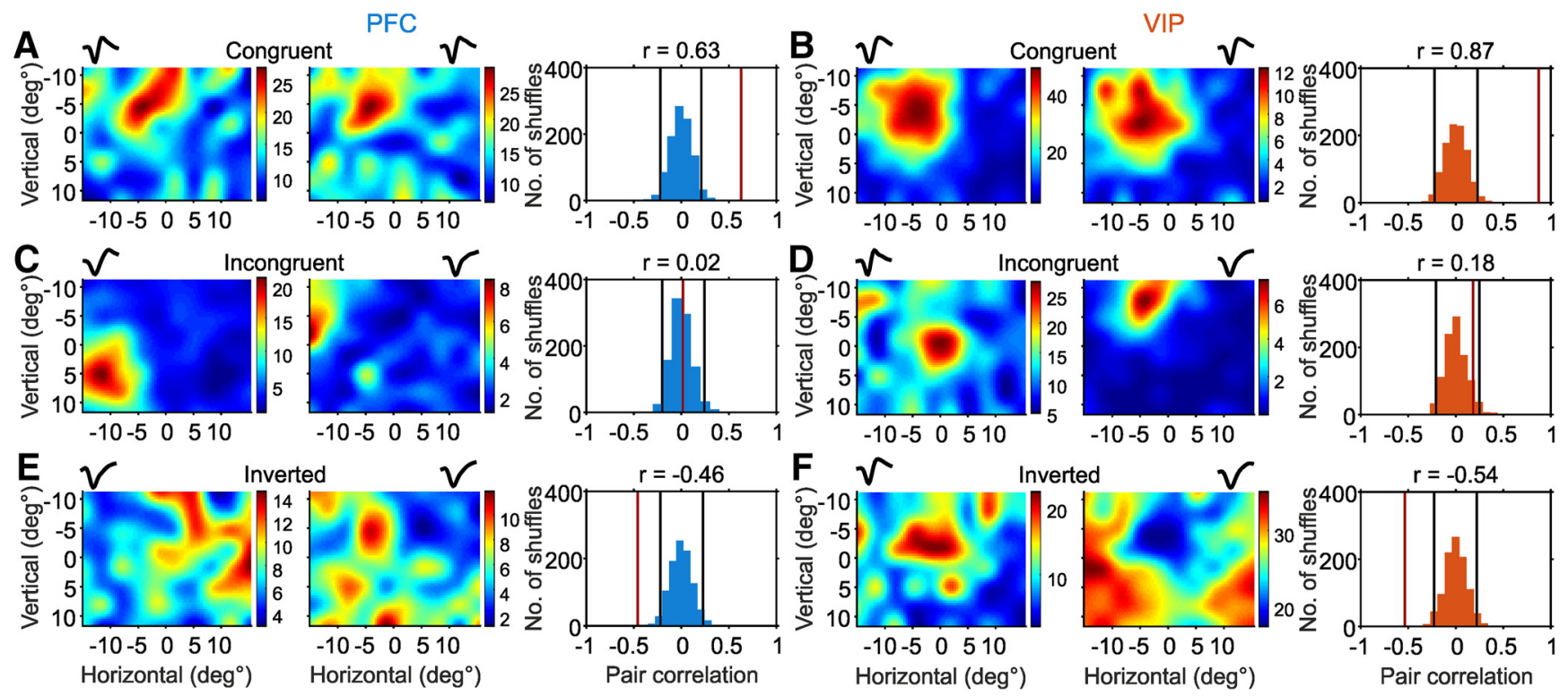

Figure 3. RFs of neuron pairs recorded on the same electrode. Left, PFC neuron pairs. Right, VIP neuron pairs. $A$, Example of PFC neuron pair recorded in Monkey L whose RF maps (left) were classified as congruent based on their $2 \mathrm{D}$ correlation coefficient, $r$ (right). The normalized averaged waveforms of these neurons are in miniature above the RF maps. Histogram of correlation coefficients obtained from shuffling the raw RF maps 1000 times and calculating the $2 \mathrm{D}$ correlation coefficient 1000 times. Black vertical lines indicate the 2.5 th and 97.5 th percentiles of the shuffled distribution. Red line indicates the true correlation coefficient obtained from the true RF maps, also stated above the histogram. As the true correlation coefficient is $>97.5$ th percentile of the data (two-tailed, $p<0.05$ ), the neuron pair was judged to have congruent RFs. $\boldsymbol{B}$, Example congruent pair from VIP. C, D, Example of an incongruent pair from each area with their corresponding correlation coefficient. Here, the true correlation coefficient lay within 2.5th and 97.5th percentiles of the shuffled distribution. $\boldsymbol{E}, \boldsymbol{F}$, Example of an inverted pair from each area. Here, the true correlations were $<2.5$ th percentile of the surrogate distribution and were negative. $\boldsymbol{B}-\boldsymbol{F}$, The neuron pairs were recorded from Monkey $\boldsymbol{S}$.

A

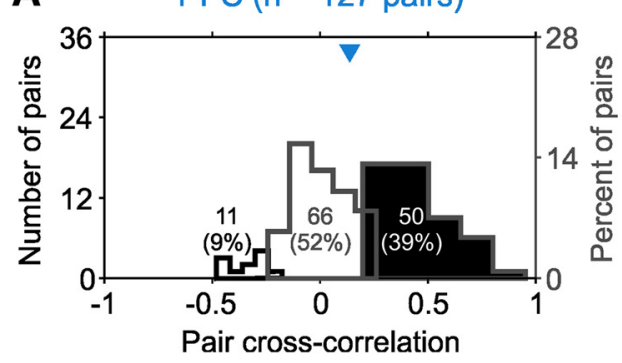

B

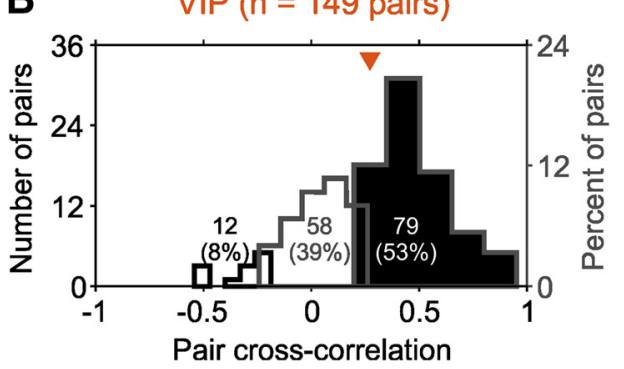

Figure 4. Heterogeneity of RFs of adjacent neurons. $\boldsymbol{A}$, Frequency distributions of inverted (white histograms with black outline), incongruent (white histograms with gray outline), and congruent neuron pairs (black histograms) from PFC. Overlaid numbers indicate the total number and percentage of pairs contained in the histograms. Colored triangles represent the medians of all the correlation coefficients. $\boldsymbol{B}$, Frequency distribution of the similarity for VIP neuron pairs. The population of recorded neuron pairs in VIP had a higher correlation coefficient than PFC (MannWhitney $U$ test, $p<0.05)$. PFC had a significantly higher frequency of incongruent pairs than congruent pairs $\left(\chi^{2}=5.33, p<0.05\right)$ compared with VIP.

kinds of RFs, we calculated a 2D correlation analysis that considered the entire raw RF map (DeAngelis et al., 1999). The true correlation coefficient for each neuron pair was then compared with a distribution of correlation coefficients derived from 1000 shuffled RF surrogates for the same neuron pair. True correlation coefficients that were significantly larger than the shuffled distribution ( $p<0.05$; positive correlation coefficient) indicated that the RFs of both neurons in the pair were congruent (Fig. $3 A, B$, right panels). True correlations that were indistinguishable from the randomly shuffled distribution indicated incongruent RFs of adjacent neurons (Fig. 3C,D, right panels). Finally, a proportion of RF pairs were significantly negatively correlated with each other ( $p<0.05$; negative correlation coefficient), and these had true correlations smaller than the shuffled distribution (Fig. $3 E, F$, right panels). We characterized this last class as inverted RF pairs. A comparison of the frequencies of $2 \mathrm{D}$ correlation coefficients across brain areas revealed that $53 \%$ of the RFs of adjacent neurons were congruent in VIP, in contrast to only $39 \%$ congru- ent RFs in PFC (Fig. 4A,B). Comparison of congruent and other RFs showed a significantly higher proportion of congruent RFs in VIP (PFC: 50 of 127, VIP: 79 of 149; $\chi^{2}=5.13, p<0.05$ ). In particular, the proportion of congruent and incongruent RFs differed significantly between the two areas (PFC: 50 of 116 congruent against 66 of 116 incongruent, VIP: 79 of 137 congruent and 58 of 137 incongruent; $\left.\chi^{2}=5.33, p<0.05\right)$. All categories are taken together; VIP neuron pairs displayed higher correlation coefficients than PFC (median $r_{2 \mathrm{D}}$ for PFC neuron pairs $=0.14$; median $r_{2 \mathrm{D}}$ for VIP neuron pairs $=0.27$; Mann-Whitney $U$ test, $z=-2.38, p<0.05)$. VIP neuron pairs, on average, were more similar than PFC neuron pairs.

We also calculated the map correlation separately for the horizontal and vertical dimensions. Along the vertical dimension, correlations between neuronal pairs in the VIP did not differ significantly from PFC pairs (median $r_{\text {vertical }}$ PFC $=0.12$, median $r_{\text {vertical }}$ VIP $=0.20$; Mann-Whitney $U$ test, $z=-1.65, p>0.05$ ). Along the horizontal dimension, however, correlations for VIP 
A

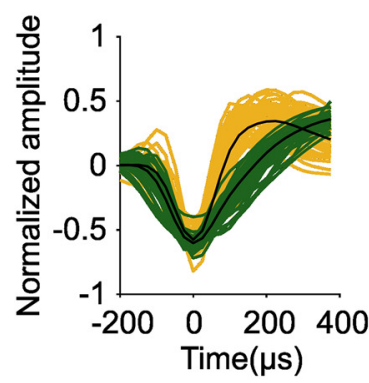

C

PFC $(n=1086$ neurons $)$

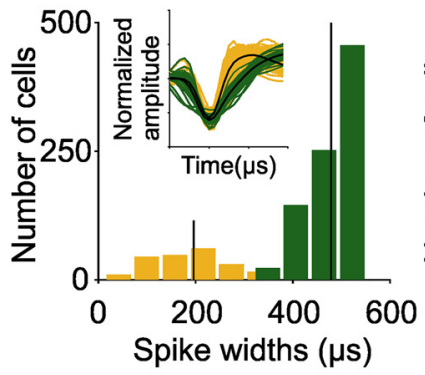

E

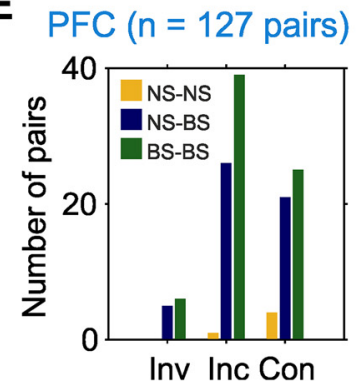

B $(n=1951$ neurons $)$

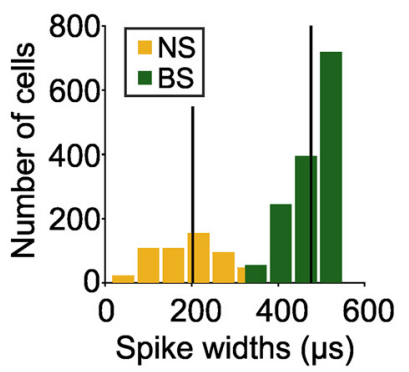

D
VIP ( $n=865$ neurons)

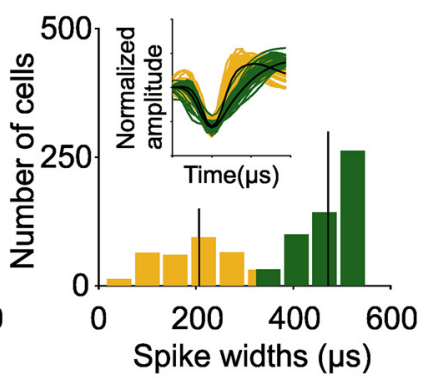

$F$

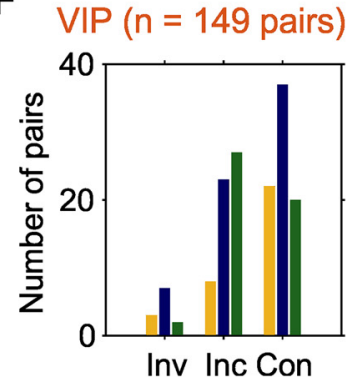

Figure 5. Classification of neuron types according to spike waveform characteristics. $\boldsymbol{A}$, Spike waveforms of 50 randomly chosen NS neurons (yellow) and BS neurons (green), aligned to their troughs. The mean waveforms of all NS and BS neurons are plotted in black. $B$, Distribution of spike widths for all NS and BS neurons. Black vertical lines indicate the widths of the average waveforms in each class. C, Histogram of 1086 PFC spike widths colored according to the classification into NS and BS classes with 50 randomly selected PFC waveforms of each class in the inset. The means of all PFC NS and BS neurons are plotted in black. Scale is the same as in A. D, Histogram of 865 VIP spike widths colored by class with 50 randomly chosen VIP waveforms of each class in the inset as in $\boldsymbol{C}$. $\boldsymbol{E}$, Distribution of RF similarity. Three classes of neuron pairs in PFC: Inv, Inverted; Inc, incongruent; Con, congruent. Yellow represents NS-NS pairs. Blue represents NS-BS pairs. Green represents BS-BS. $\boldsymbol{F}$, Same distribution as $\boldsymbol{E}$ for neuron pairs recorded in VIP.

pairs were again higher than PFC pairs (median $r_{\text {horizontal }} \mathrm{PFC}=$ 0.04 , median $r_{\text {horizontal }}$ VIP $=0.19$; Mann-Whitney $U$ test, $z=$ $-2.85, p<0.01)$.

\section{Comparing RF similarity in pairs of different cell classes}

We hypothesized that the observed heterogeneity of adjacent neurons is related to differences in the microcircuitry in association cortex. Therefore, we first classified neurons into putative inhibitory interneurons (NS) and pyramidal projection neurons (BS) based on the waveforms characteristics of their extracellular action potentials (Fig. 5A) (Swadlow and Weyand, 1987; Constantinidis and Goldman-Rakic, 2002; Johnston et al., 2009). The spike widths of all the recorded neurons so classified were well separated into two distributions (Fig. 5B). As expected based on the abundance of interneurons and projection neurons in the

neocortex (Markram et al., 2004; Shepherd, 2004), we found 81\% ( 876 of 1086) of BS and 19\% (210 of 1086) of NS neurons in PFC (Fig. 5 C). In VIP, $62 \%$ (538 of 865) of BS and 38\% (327 of 865) of NS neurons were detected (Fig. 5D). All neuron pairs were thus assigned to BS-BS, NS-BS, or NS-NS pairs. With the exception of NS-NS pairs that were almost absent in $\mathrm{PFC}, \mathrm{BS}-\mathrm{BS}(\mathrm{PFC}=70$; $\mathrm{VIP}=49)$ and NS-BS $(\mathrm{PFC}=52 ; \mathrm{VIP}=67)$ pairs were abundant in both association cortices and distributed to the congruent, incongruent, and inverted RF-pair classes according to the proportions of those RF pairs (Fig. $5 E, F$ ). We compared the proportions of congruent versus incongruent pairs in the BS-BS pairs and NS-BS pairs and found a larger proportion of NS-BS pairs in VIP to be congruent $\left(\chi^{2}=3.87, p<0.05\right)$.

\section{Functional connectivity of adjacent cell classes}

If adjacent NS and BS cells constitute elements of microcircuits operating with inhibition and excitation, their connectivity pattern might provide insights concerning the observed differences and heterogeneity in spatial tuning in association cortices. However, even juxtaposed neurons simultaneously recorded at the same electrode tip are not necessarily functionally connected and part of a local circuit. If two neurons are functionally connected, the firing of one cell systematically coincides with, or is influenced by, the activity of the other cell on a millisecond time scale. We therefore tested for functional connectivity of cell pairs based on their temporally correlated discharge patterns by applying a cross-correlation analysis to spike trains. It measures the frequency at which one cell called "target" fires relative to the firing time of a spike in another cell known as "reference" (Salinas and Sejnowski, 2001). Therefore, if two cells are functionally coupled and one cell provides inhibitory input to the other, synchronous spiking should be suppressed resulting in a negative correlation in the cross correlogram at 0 time lag. If, however, one cell provides excitatory input to the other, spiking should coincide, resulting in a positive correlation in the cross correlogram. Alternatively, cells may receive common excitatory or inhibitory input that cause broad positive or negative correlation peaks, respectively.

Although correlations between single neurons in the association cortex are typically rare (Constantinidis et al., 2001; Constantinidis and Goldman-Rakic, 2002), we found a total of 14 pairs of BS-BS and NS-BS pairs in PFC, and 15 such pairs in VIP to be significantly coupled. Functional coupling was constant across RF trials and delayed-match-to-sample trials. In the cell pairs that could be compared, 4 PFC and 4 VIP pairs remained significantly coupled and in the same directions (excitatory or inhibitory) for both trial types. Representative examples of these functionally connected neuron pairs are depicted in Figure 6 (for BS-BS pairs) and Figure 7 (for NS-BS pairs). Overall, we observed more inhibitory connections in PFC and more excitatory connections in VIP (only 3 of 14 excitatory connections in PFC compared with 13 of 15 excitatory connections in VIP, $\chi^{2}=12.46$, $p<0.001)$. In addition, the average widths of the cross correlograms were significantly broader in VIP compared with PFC (Mann-Whitney $U$ test, $p<0.05$ ).

Among BS-BS neuron pairs, we observed that 4 of 6 PFC pairs and 5 of 7 VIP were congruent (Fig. $6 A, B$ ). These pairs were connected in opposite ways in the two brain areas. Whereas the PFC pairs' correlogram showed a negative peak indicating forward inhibition (Fig. 6C), the VIP pairs showed a sharp positive peak suggesting excitatory connection (Fig. 6D). The remaining pairs were incongruent or inverted (Fig. $6 E-H$ ). Whereas all 6 BS-BS pairs in PFC shared sharp inhibitory connections (Fig. 6I), only 1 of 7 VIP BS-BS pairs shared such a connection (Fig. 6J). 
A

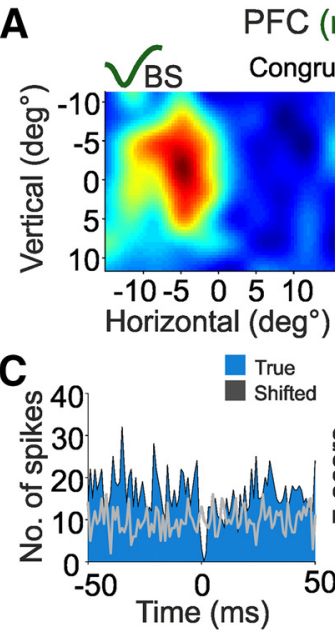

PFC ( $n=6$ pairs)
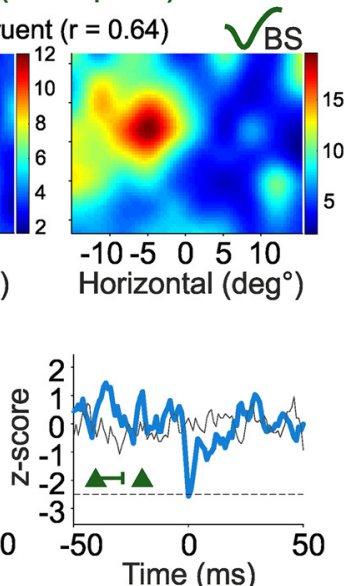

E

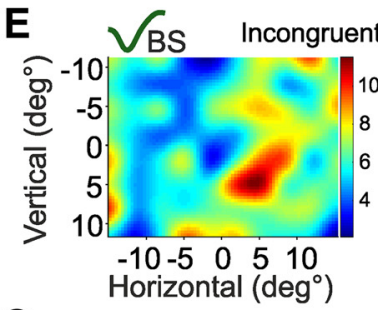

G
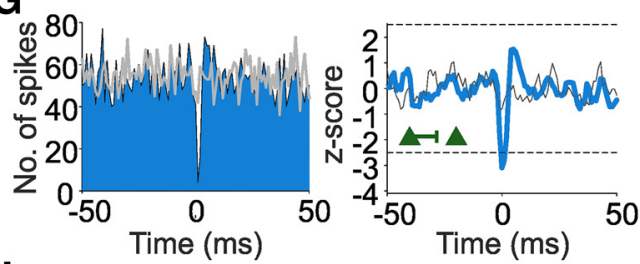

I

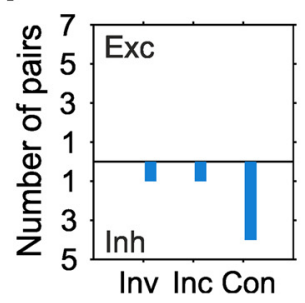

B
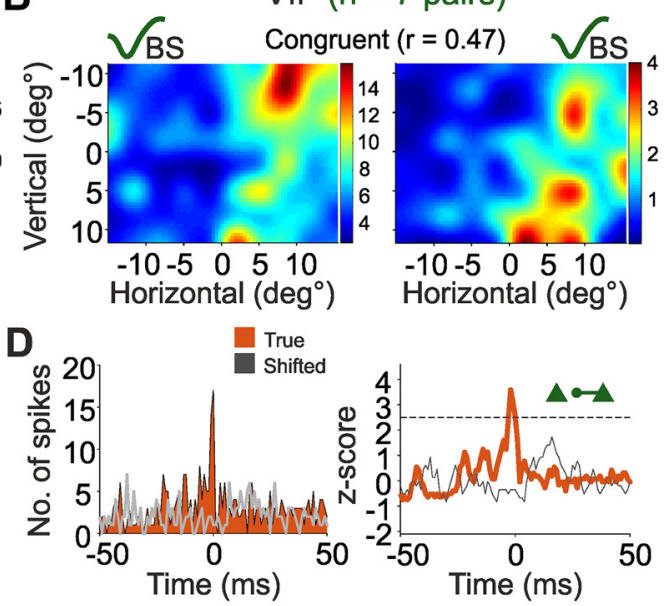

H

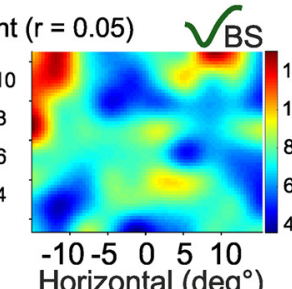

\section{J}

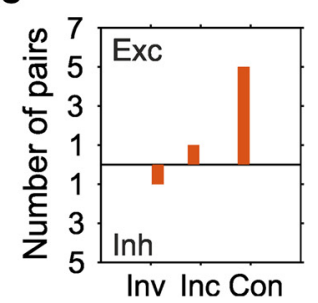

Figure 6. Distinct connectivity of BS-BS pairs in PFC and VIP. $A$, Example of a BS-BS pair with congruent RFs recorded from the same electrode in PFC. Top, Example waveforms illustrate the cell classes. The neuron used as the trigger to produce the cross-correlogram is presented on the left of each panel. $\boldsymbol{B}$, An example BS-BS pair from the VIP with congruent RFs. $\boldsymbol{C}$, Left, Raw cross-correlogram of the neuron pair in color. Gray represents the shifted cross-correlogram. The cross-correlogram shows the distribution of spike times measured in neuron from $\boldsymbol{A}$ (right) to each spike in $\boldsymbol{A}$ (left). The shifted cross-correlogram shows the distribution of spike times measured in the subsequent trial. Right, $z$ scores obtained from the raw and shifted correlograms, smoothed with a 3 ms boxcar. Dashed line indicates the significance threshold. Here, the BS neuron in $\boldsymbol{A}$ (left, top) inhibits the other BS neuron (right, top) as denoted by the green triangles linked by an inhibitory connection from left to right. $\boldsymbol{D}$, Cross-correlogram for the neuron pair presented in $\boldsymbol{B}$. Here, the BS neuron on the right is exciting the $B S$ neuron on the left as the peak has a negative lag. $\boldsymbol{E}$, An example of an incongruent BS-BS pair from PFC. $\boldsymbol{F}$, Example pair from VIP with inverted RFs. $\boldsymbol{G}$, Cross-correlograms of pair depicted in $\boldsymbol{E}$. $\boldsymbol{H}$, Cross-correlogram of pair in $\boldsymbol{F}$. Example pair $\boldsymbol{A}$ is from Monkey $L$ and the rest from Monkey S. Putative connection diagrams are shown on each correlogram. I, Summary plot of excitatory (Exc) and inhibitory (Inh) connections and the similarity index of the resultant RFs in PFC. Inv, Inverted; Inc, incongruent; Con, congruent. J, Corresponding plot for RFs in VIP.

Together, a preponderance of inhibitory connections between adjacent putative pyramidal cells in PFC contrasts with mostly excitatory connections between such cell types in VIP. Among NS-NS pairs, 12 of which we found to be functionally connected in VIP show exclusively excitatory connections, which correlated with similar RFs across those pairs ( 7 of 12 pairs were congruent). We did not find any functionally connected NS-NS pairs in PFC to compare against VIP.

For NS-BS neuron pairs, we observed 8 pairs with significant functional interaction in each area (Fig. 7). Six of these pairs were incongruent or inverted in PFC (Fig. $7 A, E, I$ ), whereas 6 of them were congruent in VIP (Fig. $7 B, F, J$ ). This difference in RF similarity of adjacent NS-BS pairs between PFC and VIP was statistically significant $\left(\chi^{2}=4, p<0.05\right)$. In NS-BS pairs of the PFC, we observed mostly sharp inhibitory ( 5 of 8 pairs) interactions $\sim 0$ time lag (Fig. $7 C, G, K)$, indicating forward inhibition of NS on BS cells. In contrast, mostly excitatory ( 7 of 8 pairs) interactions were observed for NS-BS pairs in VIP (Fig. 7D,H,L). The temporally less precise excitatory interactions in VIP with lags between -10 and 10 ms suggest common excitatory input. Inhibitory interactions between NS-BS neurons resulted in predominantly incongruent or inverted RFs (4 of 5 PFC pairs and 1 VIP pair), whereas excitatory interactions resulted in predominantly congruent RFs ( 1 of 3 PFC pairs and 6 of 7 VIP pairs) and never in inverted RFs. 
A

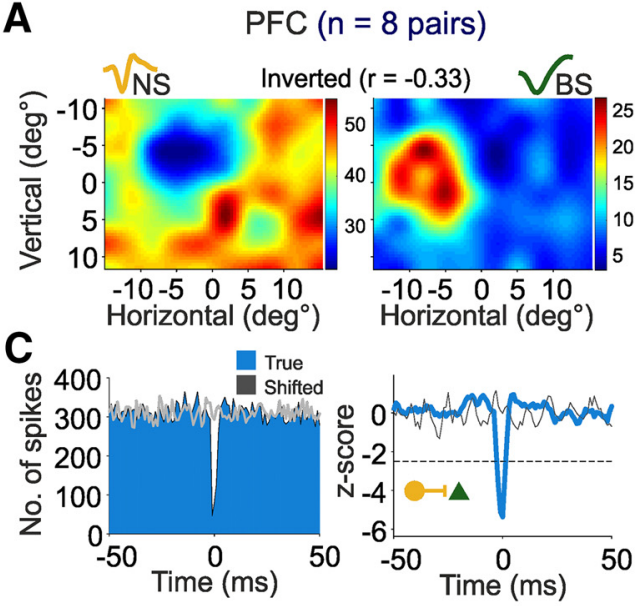

E

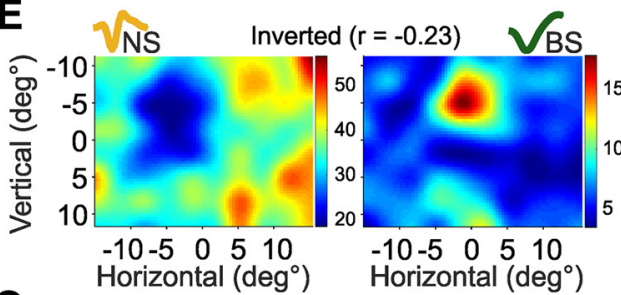

G
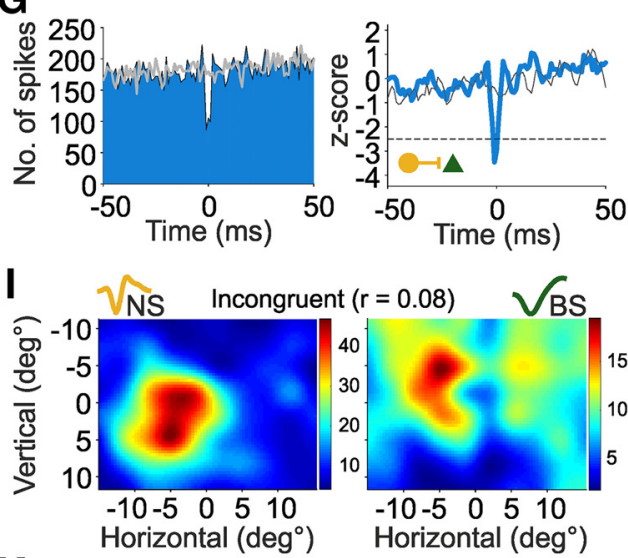

K

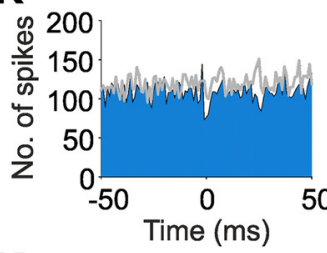

M
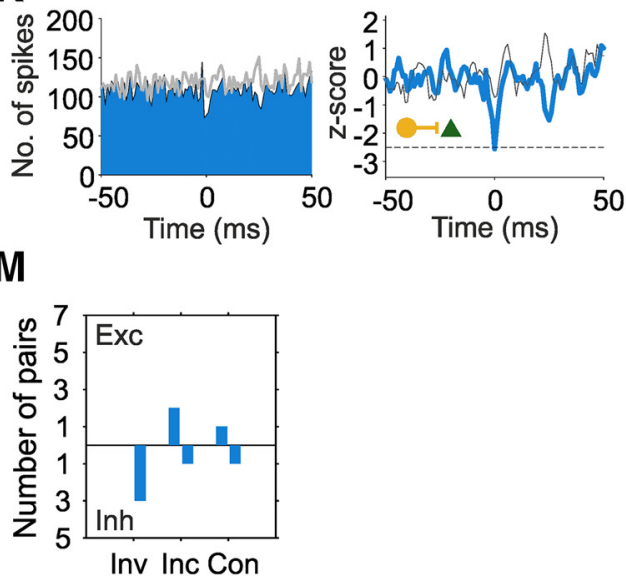

B

VIP ( $\mathrm{n}=8$ pairs)
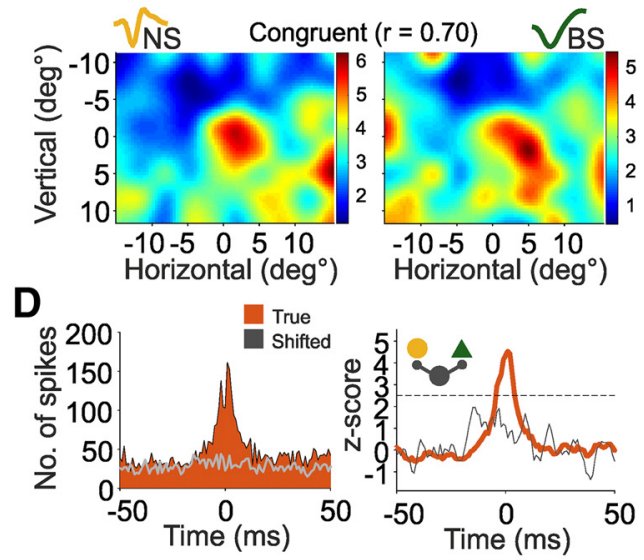

F

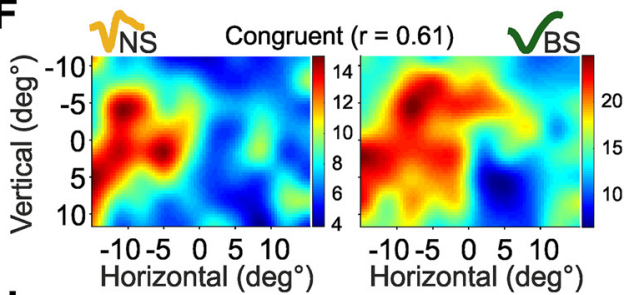

H
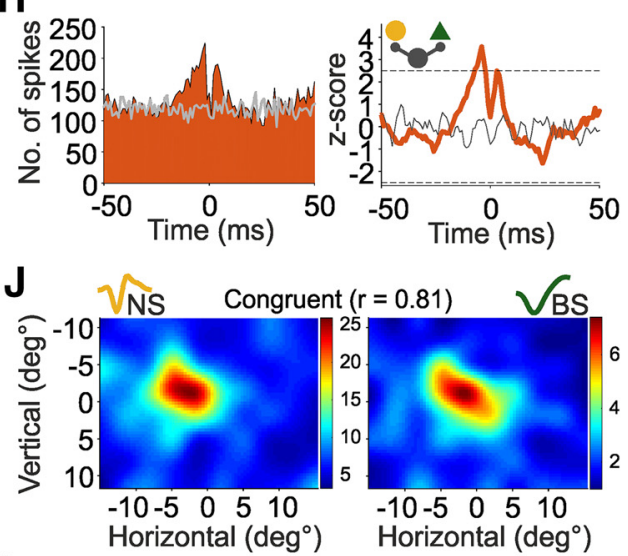

L

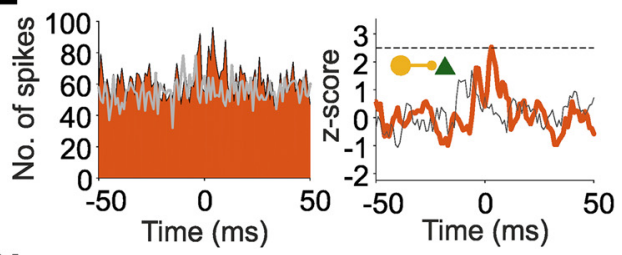

N

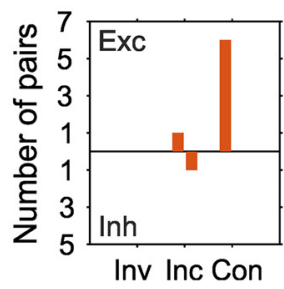

Figure 7. Distinct connectivity of NS-BS pairs in PFC and VIP.A, Example of an NS-BS pair with inverted RFs recorded from the same electrode in PFC. Same layout as in Figure 5 A, B. B, An example NS-BS pair with congruent RFs from the VIP. C, Raw cross-correlogram of the neuron pair from $\boldsymbol{A}$ (left) and z scores obtained from the correlogram in color. Gray represents the shifted correlogram. Same layout as in Figure $5 C, D$. Here, the NS neuron in $\boldsymbol{A}$ (left, above) depicted with a yellow circle inhibits the BS neuron (right, above) shown as a green triangle. $D$, Raw cross-correlogram (left) and $z$ scores of the neuron pair from $B$. Here, the NS neuron and BS neuron show a broad peak $\sim 0 \mathrm{~ms}$, indicating the presence of common excitatory input. $E$, Example NS-BS pair with inverted RFs from PFC. $\boldsymbol{F}$, Example pair from VIP with congruent RFs. $\boldsymbol{G}$, Raw cross-correlogram (left) and z scores of the neuron pair from $\boldsymbol{E}$. $\boldsymbol{H}$, Raw cross-correlogram (left) and $z$ scores of the neuron pair from $\boldsymbol{F}$. $\boldsymbol{I}$, Example PFC pair with incongruent RFs. J, Example VIP pair with congruent RFs. $\boldsymbol{K}, \boldsymbol{L}$, Raw cross-correlogram and $z$ scores of the neuron pairs from $\boldsymbol{I}$ and $\boldsymbol{J}$. Example pairs $\boldsymbol{A}, \boldsymbol{B}, \boldsymbol{E}$, and $\boldsymbol{J}$ are from Monkey L; and example pairs $\boldsymbol{F}$ and $\boldsymbol{I}$ are from Monkey S. Putative connection diagrams are shown on each correlogram. $\boldsymbol{M}, \boldsymbol{N}$, Summary plot of excitatory (Exc) and inhibitory (Inh) connections and RF similarity in PFC and VIP, respectively. Inv, Inverted; Inc, incongruent; Con, congruent. 


\section{Discussion}

We were able to demonstrate functional connectivity between adjacent neurons of extracellularly identified putative inhibitory interneurons (NS) and pyramidal cells (BS). Temporal correlations indicative of functional connectivity between adjacent single neurons in the association cortex are typically rare; only $\sim 5 \%-10 \%$ of simultaneously recorded cell pairs turn out to be functionally connected (Constantinidis et al., 2001; Constantinidis and Goldman-Rakic, 2002; Diester and Nieder, 2008). This is consistent with our findings of $\sim 10 \%$ of connected neuron pairs. Despite our large sample of simultaneously recorded cell pairs in both the PFC and the VIP, the number of connected pairs, particularly when segregated into different cell classes, remained relatively small. Despite this limitation, the differences in functional connectivity between putative inhibitory interneurons and pyramidal cells in PFC and VIP are statistically robust.

In the PFC, we found more inhibitory and also more temporally precise functional connections between adjacent neurons than in VIP. Both BS-BS and NS-BS pairs in PFC were characterized by primarily negative and sharp connections. This inhibitory connection pattern in PFC correlated with a higher frequency of incongruent or inverted RFs. Both findings are suggestive of primarily inhibitory effects of adjacent neurons in PFC. Such temporally precise inhibitory effects suggest lateral feedforward inhibition between neurons representing different visual field locations that may modulate neuronal responsiveness, especially to stimulation of the borders of RFs, effectively resulting in smaller excitatory areas. The real world does not, however, consist of single stimuli presented at a time (as in our experiment); virtually all retinal locations are affected simultaneously. This multitude of competing activations at different locations may cause strong inhibitory interactions between neurons located at different points of the visuotopic map and perhaps a substantial shrinkage of excitatory RFs toward their functional center. Lateral inhibition may contribute to the production of such sharp peaks of neural activity as a function of location in the visual field.

The functional connectivity of cell types with respect to the properties of spatial RFs has not been investigated in PFC (or VIP) before. However, it has been shown that neighboring NS and BS cells in the primate PFC can exhibit opposite tuning characteristics. When comparing the responses of NS and BS neurons that were recorded within $400 \mathrm{~mm}$ of each other, Wilson et al. (1994) observed opposite spatial location selectivity of NS and BS cells (but see also Rao et al., 1999 for similar spatial preferences of adjacent NS and BS neurons). Moreover, inhibitory connections were predominant in PFC cell pairs with dissimilar spatial tuning profiles (Constantinidis and Goldman-Rakic, 2002). Beyond sculpting the processing of spatial information, inhibitory input by putative interneurons has also been implicated in shaping the tuning to numerosities of putative PFC pyramidal cells (Diester and Nieder, 2008; Nieder, 2016) and tuning to objects of nearby neurons in the IT cortex (Wang et al., 2000, 2002; Tamura et al., 2004). Because different cortical cell types also show distinct sensitivity to dopaminergic influence (Jacob et al., 2013, 2016; Ott et al., 2014), the shaping of RFs may also be modulated by neuromodulators, in addition to GABAergic inhibition.

The functional connectivity patterns in relation to cell types differed markedly in parietal area, VIP. We found more excitatory and temporally less precise connections in VIP relative to PFC. Both BS-BS and NS-BS neuron pairs showed excitatory connections. However, whereas BS-BS pairs exhibited sharp positive correlation peaks indicative of excitatory feedforward excitation,
NS-BS pairs mostly showed broad excitatory peaks suggesting common input. This is reminiscent of V1, where fast-spiking interneurons and neighboring pyramidal neurons have been found to share excitatory input (Yoshimura and Callaway, 2005; Yoshimura et al., 2005). Consistent with the idea of common input is the finding of mostly congruent RFs of NS-BS pairs. This points to a V1-like response pooling within a certain local population (Swadlow and Gusev, 2002). These remarkable differences in the functional connectivity of adjacent cortical cell types between PFC and VIP argue for substantial differences in the wiring of posterior parietal cortex that shares patterns with early visual areas, and prefrontal local circuits that seem to have developed differently.

The observed incongruencies in spatial RF characteristics and functional connectivity argue for differences in microcolumn organization between neocortical areas that subserve different functions. The reciprocally connected association areas of the parietal and frontal cortices are particularly well suited to maintain information across time and to exert cognitive control (Miller and Cohen, 2001). In contrast to early visual cortices, the areas of the posterior parietal and prefrontal cortices are connected not only to each other (Lewis and Van Essen, 2000), but also up to over a dozen other widely distributed cortical areas, all interconnected by common thalamic input from the medial pulvinar nucleus (Selemon and Goldman-Rakic, 1988). In addition, the areas in this network display noncanonical circuit properties (Goldman-Rakic, 1988); in contrast to canonical circuits of the sensory and motor cortices, many connections within this network lack a clear sensory-motor hierarchical polarity with consistent feedforward and feedback laminar termination patterns. Instead, the noncanonical network seems to be designed for parallel and reentrant processing. Such recurrent loops of the noncanonical association cortex give rise to persistent (or sustained) neuronal activity that enables neurons to actively buffer and process information during working memory periods (Merten and Nieder, 2012). Because high-level cognitive functioning would be impossible without persistent activity, microcircuits and their excitatory and inhibitory neuronal connections might be geared toward reentrant loops and, therefore, inevitably differ from sensory neocortex.

\section{References}

Aertsen AM, Gerstein GL, Habib MK, Palm G (1989) Dynamics of neuronal firing correlation: modulation of "effective connectivity." J Neurophysiol 61:900-917.

Avillac M, Denève S, Olivier E, Pouget A, Duhamel JR (2005) Reference frames for representing visual and tactile locations in parietal cortex. Nat Neurosci 8:941-949. CrossRef Medline

Avillac M, Ben Hamed S, Duhamel JR (2007) Multisensory integration in the ventral intraparietal area of the macaque monkey. J Neurosci 27: 1922-1932. CrossRef Medline

Chen X, DeAngelis GC, Angelaki DE (2014) Eye-centered visual receptive fields in the ventral intraparietal area. J Neurophysiol 112:353-361. CrossRef Medline

Colby CL, Duhamel JR, Goldberg ME (1993) Ventral intraparietal area of the macaque: anatomic location and visual response properties. J Neurophysiol 69:902-914. Medline

Constantinidis C, Goldman-Rakic PS (2002) Correlated discharges among putative pyramidal neurons and interneurons in the primate prefrontal cortex. J Neurophysiol 88:3487-3497. CrossRef Medline

Constantinidis C, Franowicz MN, Goldman-Rakic PS (2001) Coding specificity in cortical microcircuits: a multiple-electrode analysis of primate prefrontal cortex. J Neurosci 21:3646-3655. Medline

DeAngelis GC, Ghose GM, Ohzawa I, Freeman RD (1999) Functional micro-organization of primary visual cortex: receptive field analysis of nearby neurons. J Neurosci 19:4046-4064. Medline 
de Oliveira SC, Thiele A, Hoffmann KP (1997) Synchronization of neuronal activity during stimulus expectation in a direction discrimination task. J Neurosci 17:9248-9260. Medline

Diester I, Nieder A (2008) Complementary contributions of prefrontal neuron classes in abstract numerical categorization. J Neurosci 28:77377747. CrossRef Medline

Douglas RJ, Martin KA (2004) Neuronal circuits of the neocortex. Annu Rev Neurosci 27:419-451. CrossRef Medline

Duhamel JR, Colby CL, Goldberg ME (1998) Ventral intraparietal area of the macaque: congruent visual and somatic response properties. J Neurophysiol 79:126-136. Medline

Fuster JM (2000) Executive frontal functions. Exp Brain Res 133:66-70. CrossRef Medline

Goldman-Rakic PS (1988) Topography of cognition: parallel distributed networks in primate association cortex. Annu Rev Neurosci 11:137-156. CrossRef Medline

Goldman-Rakic PS (1995) Cellular basis of working memory. Neuron 14: 477-485. CrossRef Medline

Guipponi O, Wardak C, Ibarrola D, Comte JC, Sappey-Marinier D, Pinède S, Ben Hamed S (2013) Multimodal convergence within the intraparietal sulcus of the macaque monkey. J Neurosci 33:4128-4139. CrossRef Medline

Hubel DH, Wiesel TN (1962) Receptive fields, binocular interaction and functional architecture in the cat's visual cortex. J Physiol 160:106-154. CrossRef Medline

Jacob SN, Nieder A (2014) Complementary roles for primate frontal and parietal cortex in guarding working memory from distractor stimuli. Neuron 83:226-237. CrossRef Medline

Jacob SN, Ott T, Nieder A (2013) Dopamine regulates two classes of primate prefrontal neurons that represent sensory signals. J Neurosci 33:1372413734. CrossRef Medline

Jacob SN, Stalter M, Nieder A (2016) Cell-type-specific modulation of targets and distractors by dopamine D1 receptors in primate prefrontal cortex. Nat Commun 7:13218. CrossRef Medline

Johnston K, DeSouza JF, Everling S (2009) Monkey prefrontal cortical pyramidal and putative interneurons exhibit differential patterns of activity between prosaccade and antisaccade tasks. J Neurosci 29:5516-5524. CrossRef Medline

Kravitz DJ, Saleem KS, Baker CI, Mishkin M (2011) A new neural framework for visuospatial processing. Nat Rev Neurosci 12:217-230. CrossRef Medline

Kremkow J, Jin J, Wang Y, Alonso JM (2016) Principles underlying sensory map topography in primary visual cortex. Nature 533:52-57. CrossRef Medline

Lewis JW, Van Essen DC (2000) Corticocortical connections of visual, sensorimotor, and multimodal processing areas in the parietal lobe of the macaque monkey. J Comp Neurol 428:112-137. CrossRef Medline

Markram H, Toledo-Rodriguez M, Wang Y, Gupta A, Silberberg G, Wu C (2004) Interneurons of the neocortical inhibitory system. Nat Rev Neurosci 5:793-807. CrossRef Medline

Merchant H, de Lafuente V, Peña-Ortega F, Larriva-Sahd J (2012) Functional impact of interneuronal inhibition in the cerebral cortex of behaving animals. Prog Neurobiol 99:163-178. CrossRef Medline

Merten K, Nieder A (2012) Active encoding of decisions about stimulus absence in primate prefrontal cortex neurons. Proc Natl Acad Sci U S A 109:6289-6294. CrossRef Medline

Mikami A, Ito S, Kubota K (1982) Visual response properties of dorsolateral prefrontal neurons during visual fixation task. J Neurophysiol 47:593605. Medline

Miller EK, Cohen JD (2001) An integrative theory of prefrontal cortex function. Annu Rev Neurosci 24:167-202. CrossRef Medline

Mishkin M, Ungerleider LG, Macko KA (1983) Object vision and spatial vision: two cortical pathways. Trends Neurosci 6:414-417. CrossRef

Nieder A (2012) Supramodal numerosity selectivity of neurons in primate prefrontal and posterior parietal cortices. Proc Natl Acad Sci U S A 109: 11860-11865. CrossRef Medline

Nieder A (2016) The neuronal code for number. Nat Rev Neurosci 17:366382. CrossRef Medline

Nieder A, Miller EK (2004) A parieto-frontal network for visual numerical information in the monkey. Proc Natl Acad Sci U S A 101:7457-7462. CrossRef Medline

O'Scalaidhe SP, Wilson FA, Goldman-Rakic PS (1997) Areal segregation of face-processing neurons in prefrontal cortex. Science 278:1135-1138. CrossRef Medline

Ott T, Jacob SN, Nieder A (2014) Dopamine receptors differentially enhance rule coding in primate prefrontal cortex neurons. Neuron 84:13171328. CrossRef Medline

Rainer G, Asaad WF, Miller EK (1998) Memory fields of neurons in the primate prefrontal cortex. Proc Natl Acad Sci U S A 95:15008-15013. CrossRef Medline

Ramirez-Cardenas A, Moskaleva M, Nieder A (2016) Neuronal representation of numerosity zero in the primate parieto-frontal number network. Curr Biol 26:1285-1294. CrossRef Medline

Rao SG, Williams GV, Goldman-Rakic PS (1999) Isodirectional tuning of adjacent interneurons and pyramidal cells during working memory: evidence for microcolumnar organization in PFC. J Neurophysiol 81:19031916. Medline

Romero MC, Janssen P (2016) Receptive field properties of neurons in the macaque anterior intraparietal area. J Neurophysiol 115:1542-1555. CrossRef Medline

Rosa MG (1997) Cerebral cortex, Vol 12: Extrastriate cortex in primates (Rockland KS, Kaas JH, Peters A, eds). New York: Plenum.

Salinas E, Sejnowski TJ (2001) Correlated neuronal activity and the flow of neural information. Nat Rev Neurosci 2:539-550. CrossRef Medline

Schlack A, Sterbing-D'Angelo SJ, Hartung K, Hoffmann KP, Bremmer F (2005) Multisensory space representations in the macaque ventral intraparietal area. J Neurosci 25:4616-4625. CrossRef Medline

Selemon LD, Goldman-Rakic PS (1988) Common cortical and subcortical targets of the dorsolateral prefrontal and posterior parietal cortices in the rhesus monkey: evidence for a distributed neural network subserving spatially guided behavior. J Neurosci 8:4049-4068. Medline

Shepherd GM (2004) The synaptic organization of the brain, Ed 5. New York: Oxford UP

Sugihara T, Diltz MD, Averbeck BB, Romanski LM (2006) Integration of auditory and visual communication information in the primate ventrolateral prefrontal cortex. J Neurosci 26:11138-11147. CrossRef Medline

Suzuki H, Azuma M (1983) Topographic studies on visual neurons in the dorsolateral prefrontal cortex of the monkey. Exp Brain Res 53:47-58. Medline

Swadlow HA, Gusev AG (2002) Receptive-field construction in cortical inhibitory interneurons. Nat Neurosci 5:403-404. CrossRef Medline

Swadlow HA, Weyand TG (1987) Corticogeniculate neurons, corticotectal neurons, and suspected interneurons in visual cortex of awake rabbits: receptive-field properties, axonal properties, and effects of EEG arousal. J Neurophysiol 57:977-1001. Medline

Tamura H, Kaneko H, Kawasaki K, Fujita I (2004) Presumed inhibitory neurons in the macaque inferior temporal cortex: visual response properties and functional interactions with adjacent neurons. J Neurophysiol 91:2782-2796. CrossRef Medline

Viswanathan P, Nieder A (2013) Neuronal correlates of a visual "sense of number" in primate parietal and prefrontal cortices. Proc Natl Acad Sci U S A 110:11187-11192. CrossRef Medline

Viswanathan P, Nieder A (2015) Differential impact of behavioral relevance on quantity coding in primate frontal and parietal neurons. Curr Biol 25:1259-1269. CrossRef Medline

Wang Y, Fujita I, Murayama Y (2000) Neuronal mechanisms of selectivity for object features revealed by blocking inhibition in inferotemporal cortex. Nat Neurosci 3:807-813. CrossRef Medline

Wang Y, Fujita I, Tamura H, Murayama Y (2002) Contribution of GABAergic inhibition to receptive field structures of monkey inferior temporal neurons. Cereb Cortex 12:62-74. CrossRef Medline

Wilson FA, O'Scalaidhe SP, Goldman-Rakic PS (1994) Functional synergism between putative gamma-aminobutyrate-containing neurons and pyramidal neurons in prefrontal cortex. Proc Natl Acad Sci U S A 91: 4009-4013. CrossRef Medline

Yoshimura Y, Callaway EM (2005) Fine-scale specificity of cortical networks depends on inhibitory cell type and connectivity. Nat Neurosci 8:1552-1559. CrossRef Medline

Yoshimura Y, Dantzker JL, Callaway EM (2005) Excitatory cortical neurons form fine-scale functional networks. Nature 433:868-873. CrossRef Medline

Zaksas D, Pasternak T (2006) Directional signals in the prefrontal cortex and in area MT during a working memory for visual motion task. J Neurosci 26:11726-11742. CrossRef Medline 